\title{
Modeling the Physiological Mechanisms of Multistability in Spontaneous Corticothalamic Dynamics
}

\author{
Joost X. Maier ${ }^{1}$ and Rikkert Hindriks ${ }^{2}$ \\ ${ }^{1}$ Department of Psychology, Brandeis University, Waltham, Massachusetts 02454 and ${ }^{2}$ Department of Clinical Neurophysiology, University of Twente, 7500 \\ AE Enschede, The Netherlands \\ Review of Freyer et al.
}

Brains function through interactions between millions of neurons distributed across many different cortical and subcortical areas. These interactions result in global activity patterns that can be measured using noninvasive electrophysiological techniques in humans, such as EEG. To bridge the gap between electrophysiological processes that take place at the level of single neurons and the large scale processes that are reflected in the EEG, mean field models are employed. Neural mean field models are constructed by averaging microscopic variables (e.g., membrane potentials) over pieces of neural tissue. The idea behind this approach is that the activity of an individual neuron is not effective in influencing a target area unless its activity is paralleled by many other neurons; the so-called mass action principle (Freeman, 1975). The averaged variables in mean field models reflect this common activity of local neuronal populations and are commonly used as a model for measures of large-scale neural activity (Deco et al., 2008).

One particularly prominent feature of the EEG is the alpha rhythm. The term alpha rhythm refers to periodic fluctuations of the electrophysiological signal between 8 and $12 \mathrm{~Hz}$ and was first observed

Received May 27, 2011; revised June 27, 2011; accepted June 28, 2011.

Correspondence should be addressed to Joost X. Maier, Department of

Psychology, Brandeis University, 415 South Street, MS 062, Waltham, MA 02454. E-mail: joost.maier@gmail.com.

DOI:10.1523/JNEUROSCI.2656-11.2011

Copyright $\odot 2011$ the authors $\quad 0270-6474 / 11 / 3111423-02 \$ 15.00 / 0$ over 80 years ago (Berger, 1929). While behavioral and cognitive correlates of the alpha rhythm in humans are well documented, there is still no consensus on the biophysical mechanisms behind its generation or on its dynamics. Some researchers claim that the alpha rhythm is generated by so-called pacemaker cells, located either in the cortex or in thalamic nuclei, and propagates from there to other structures. Others suggest that no single group of cells is responsible for the generation of the alpha rhythm, but instead it emerges through interactions between distributed neural populations in cortex and/or thalamus. Accordingly, initial mean field modeling of the alpha rhythm concentrated on distributed neural populations within these structures.

A new direction was initiated with the model proposed by Rennie et al. (2002) and developed in subsequent studies in which both cortex and thalamus were included. According to this model, which forms the basis for a recent paper by Freyer et al. (2011), cortex and thalamus are individually incapable of sustaining alpha activity. Instead, the alpha rhythm emerges from the reciprocal connections between cortex and thalamus.

Work over the past 20 years has focused on whether the dynamics of the alpha rhythm are linear or nonlinear. In the context of dynamical systems, linearity and nonlinearity correspond to fixed-point and limit-cycle dynamics, respectively. In systems exhibiting fixed-point dynamics, oscillations emerge through a passive mechanism so that oscillations are produced only when the system receives external input. In contrast, in systems exhibiting limit-cycle dynamics, oscillations emerge and are sustained within the system in an active fashion, even in the absence of input. Although most experimental evidence pointed toward fixed-point dynamics in the alpha rhythm, some evidence was found for the existence of limit-cycle dynamics (Stam, 2005). The study by Freyer et al. (2011) resolves these apparently conflicting views by demonstrating that the EEG alpha rhythm is bistable: it constantly switches between fixed-point and limit-cycle dynamics under the influence of stochastic input.

By augmenting a previously developed mean field model of the thalamocortical circuit, the authors propose a biophysical mechanism that can explain the dynamics of amplitude fluctuations observed in the human EEG (Freyer et al., 2009). The proposed mechanism contains two components. First, in addition to a "waxing and waning" rhythm, which reflects the dynamics of the thalamocortical circuit around a stable fixed point, the authors hypothesize that bistability of amplitude fluctuations is caused by the vicinity of a limit cycle. Specifically, due to stochastic fluctuations in the dendritic trees of thalamocortical relay cells, the thalamocortical circuit switches erratically between a fixed point and a limit cycle. The low- and highamplitude power modes in the EEG correspond to fixed-point and limit-cycle 
dynamics, respectively. Using bifurcation theory, the authors show that the transition between these two modes is a socalled subcritical Hopf bifurcation-a dynamical mechanism through which high-amplitude oscillations can emerge. Using model simulations, they further show that the proposed mechanism quantitatively agrees with empirical EEG data in two respects: (1) the standard deviation of each power mode scales in proportion to its mean; and (2) the distribution of time spent in the two activity modes follows a long-tailed, stretched exponential form. Second, while stochastic fluctuations can explain the experimentally observed bistable behavior, its exact statistics [characterized by (1) and (2) above] could only be explained by assuming a dependence of the strength of the stochastic fluctuations on the feedback from cortex to thalamocortical relay cells. Freyer et al. (2011) speculate that such dependence could be mediated by the effective gating of NMDA receptors by AMPA receptors.

The work described above provides a biophysical explanation for multistability of the alpha rhythm in the EEG of healthy humans at rest. Multistable dynamics in large-scale neuronal networks appear to be a widespread phenomenon and is therefore of great interest. The strength of the approach taken by Freyer et al. (2011) lies in the use of a physiologically inspired mean field model. The use of such models allows direct inferences concerning the biophysical mechanisms underlying the observed phenomena and are greatly enhancing our understanding of brain function and dysfunction. Many disorders of the nervous system, both neurological (e.g., epilepsy, dementia) and psychiatric (e.g., psychosis, schizophrenia) are characterized by abnormal large-scale neural dynamics. Mean field modeling of these dynamics will provide detailed insight into the physiology that causes these disorders.

Large-scale neural dynamics are also thought to play an important role in normal brain function. However, much of the work, including the work by Freyer et al. (2011) focuses on "spontaneous" activity patterns (i.e., signals collected in the absence of a controlled context). This leaves the question of how multistable neural dynamics are paralleled by dynamics in perception or behavior untouched. Over the past years, approaches using simultaneous monitoring of large-scale neural activity in specific task contexts have already provided some insight into the nature of brain-behavior interactions (Varela et al., 2001). Mean field modeling provides an excellent framework for advancing our understanding of such interactions because it is grounded in physiologically plausible processes. Embedding neural mean field models to include environmental and/or behavioral parameters will allow the study of how specific neurophysiological processes are constrained by behavioral and environmental parameters and vice versa.

\section{References}

Berger H (1929) Über das Elektroenkephalogramm des Menschen. Arch Psychiatr Nervenkr 87:527-570.

Deco G, Jirsa VK, Robinson PA, Breakspear M, Friston K (2008) The dynamic brain: from spiking neurons to neural masses and cortical fields. PLoS Comput Biol 4:e1000092.

Freeman W (1975) Mass action in the nervous system. New York: Academic.

Freyer F, Aquino K, Robinson PA, Ritter P, Breakspear M (2009) Bistability and non-Gaussian fluctuations in spontaneous cortical activity. J Neurosci 29:8512-8524.

Freyer F, Roberts JA, Becker R, Robinson PA, Ritter P, Breakspear M (2011) Biophysical mechanisms of multistability in resting-state cortical rhythms. J Neurosci 31:6353-6361.

Rennie CJ, Robinson PA, Wright JJ (2002) Unified neurophysical model of EEG spectra and evoked potentials. Biol Cybern 86:457-471.

Stam CJ (2005) Nonlinear dynamical analysis of EEG and MEG: review of an emerging field. Clin Neurophysiol 116:2266-2301.

Varela F, Lachaux JP, Rodriguez E, Martinerie J (2001) The brainweb: phase synchronization and large-scale integration. Nat Rev Neurosci 2:229-239. 